\title{
The QT Interval and Predicting Risk in Toxicology
}

\author{
Ingrid Berling ${ }^{1,2,3}$
}

Received: 16 August 2021 / Revised: 21 August 2021 / Accepted: 21 August 2021 / Published online: 31 August 2021

(c) Crown 2021

As clinical toxicologists, much of our role is early risk assessment of the patient to predict potential bad outcomes and intervene to minimise morbidity, mortality, or costs. Not only must we predict risk, but we must also be wise stewards of the health care system and balance any potential risk with the use of hospital resources, facilities, patient time, cost, and impact. Cardiac toxicity and interpretation of electrocardiogram (ECG) intervals is one of our expert core skills which we must maintain and adapt as new scientific data comes along. The study by Shastry et al. in JMT draws an association between delayed QT interval prolongation and adverse cardiovascular events [1]. Their study importantly highlights the QT interval as a risk assessment tool and draws attention to the question of ECG timing in predicting risk. As the authors openly acknowledge in their limitations section, there are challenges with performing this type of study, particularly a secondary analysis of data, and Shastry et al. provide readers with appropriate caution on interpretating their results. The lack of certain data, such as ingestion details and the non-standardised timing of the initial and follow-up ECG, may limit the extension of their findings into clinical practice. Also candidly highlighted by the investigators is their acknowledgment that association does not necessarily amount to causation. There may be risk in using the QT interval to predict outcomes that do not have a direct pathophysiological relationship. For instance, associating shock or myocardial injury, as evidenced by troponin elevation, to a delayed QT interval does not reflect the underlying mechanisms of risk associated with the QT interval

Supervising Editor: Mark B. Mycyk, MD

Ingrid Berling

Ingrid.berling@gmail.com

1 Department of Clinical Toxicology and Pharmacology, Calvary Mater Newcastle, Newcastle, NSW, Australia

2 School of Medicine and Public Health, University of Newcastle, Newcastle, NSW, Australia

3 New South Wales Poisons Information Centre, Sydney, NSW, Australia prolongation. Likewise, linking a drug that is unlikely to cause QT interval prolongation to delayed ECG changes that have presumably been caused by other factors may create a misunderstanding and could result in drugs being labelled at risk of causing QT interval prolongation when they do not. Finally, there is also continued debate regarding which is the best method of measurement of the QT interval, and certainly the use of the ECG measured QTc with its own limitations should be noted, as we will discuss below.

The measurement of the QT interval on the ECG is used as a risk assessment tool for predicting Torsade de Pointes (TdP) and other cardiac dysrhythmias. TdP is a rare phenomenon, even in patients who have intentionally self-poisoned [2]. Physiologically, the QT interval represents the repolarisation stage (stage III) of the cardiac action potential, such that lengthening of the interval (a long QT) signifies a delayed repolarisation phase, usually due to potassium channel blockade. This elongated action potential has an increased absolute refractory period and a more positively charged state. This occurs with variation across the heart (increased QT dispersion) between epicardium and myocardium for instance. During this prolonged phase, L-type calcium channels can reactivate and early afterdepolarisations (EADs) occur which can trigger a re-entry phenomenon and proceed to episodes of TdP [3]. Thus, for TdP to occur, multiple successive events must be present, even when the QT interval is long. This chain of circumstances explains both why TdP is rare and also why the QT interval is not the ideal risk prediction tool.

Despite its shortcomings, the QT interval is currently the best assessment tool we have for predicting TdP, or perhaps more correctly, excluding TdP risk. Correctly interpreted in context, a normal ECG on presentation can remove a patient from a lengthy observation period on cardiac monitoring. However, we do know the QT interval is not static nor are the dynamic effects of poisonings. It changes with heart rate, time of day, electrolyte shifts, drug concentrations, and countless other yet to be described factors. The need for further screening or monitoring must be guided by our knowledge of which drugs are at risk of prolonging the QT 
and what factors influence the onset, duration, and severity of that prolongation. Unless interpreted in these specific and dynamic contexts, we risk over assessing, over monitoring, and over treating. For instance, we can deduct that the timing of QT interval changes will be related to differences in drug absorption and distribution, potential active metabolites, elimination half-life, co-ingestants, pharmacokinetic and pharmacodynamic drug interactions, and individual factors including electrolyte imbalances and comorbidities. For example, previous modelling work on citalopram ingestions demonstrated that QT interval prolongation is delayed [4], and this generated a recommendation for 12 hours of cardiac monitoring post ingestion of more than $600 \mathrm{mg}[5,6]$. Equally, we know benzodiazepines, even in overdose, are not at risk of causing QT interval prolongation [7, 8].

Limitations in using the ECG printout data, rather than visual interpretation of the ECG morphology, are paramount to take into account. It is known that standard ECG algorithms cannot accurately measure the QT interval, particularly when the $\mathrm{T}$ wave is long or the morphology is unusual [9]. Under-reporting of the QT interval by the ECG machine occurs. Raw manual measurement of the QT interval and its heart rate pair is advocated $[10,11]$. This is because the QT interval is directly related to the length of the cardiac cycle. If a patient has a fast heart rate, they will have a shortened cardiac cycle or time in which to complete each cardiac action potential, and also less time to trigger EADs (sometimes referred to as protective). The slower the heart rate the longer the cardiac cycle and longer the QT interval can become. Mathematical formulae, whether programmed in ECG machines or calculated by hand, such as Bazett's, that aim to correct the QT interval into a standardised corrected QT (QTc) for heart rate, routinely over-correct in tachycardia and under-correct in bradycardia[12]. Quetiapine is a classic example of a drug that results in an ECG machine long QTc reading due to tachycardia when manual review of the ECG shows no raw QT prolongation [13].

Shastry et al. raise the issue of delayed QT interval prolongation even when the initial presentation ECG may be normal; however, the question of who to monitor and for how long remains relatively unanswered. Monitoring patients who are not at risk of delayed QT changes places a burden on our health care system. As the authors acknowledge, we must be cautious in drawing any conclusions made from these data given the limitations discussed, and rather use the recognition of the changing QT interval over time to further drive research on the QT interval in drug overdose. Collaborative and prospective research should include ingestion details, context and timing of the ECG with visual ECG interpretation, in the context of heart rate, to correlate findings with drug pharmacodynamic and kinetic data to develop evidence-based guidelines such as those established for citalopram overdose in Australia [5]. Research should focus on drugs with a physiological mechanism for possible delayed
QT changes. Meanwhile, astute clinical assessment, attention to patient vital signs, monitoring of electrolytes, and visual interpretation of the ECG in the context of ingestion details remain our most useful predictors of potential cardiovascular events.

Sources of Funding None.

\section{Declarations}

Conflicts of Interest None.

\section{References}

1. Shastry S, Aluise ER, Richardson LD, Vedanthan R, Manini AF. Delayed QT prolongation: derivation of a novel risk factor for adverse cardiovascular events from acute drug overdose. J Med Toxicol. https://doi.org/10.1007/s13181-021-00855-2.

2. Al-Abri SA, Woodburn C, Olson KR, Kearney TE. Ventricular dysrhythmias associated with poisoning and drug overdose: a 10-year review of statewide poison control center data from California. Am J Cardiovasc Drugs. 2015;15(1):43-50.

3. Roden DM. Cellular basis of drug-induced torsades de pointes. $\mathrm{Br}$ J Pharmacol. 2008;154(7):1502-7.

4. Friberg LE, Isbister GK, Duffull SB. Pharmacokinetic-pharmacodynamic modelling of QT interval prolongation following citalopram overdoses. Br J Clin Pharmacol. 2006;61(2):177-90.

5. eTG complete. Melbourne: therapeutic guidelines limited; 2020. Available on CIAP. https://www.tg.org.au. Accessed 29 Aug 2021.

6. Isbister GK, Friberg LE, Duffull SB. Application of pharmacokinetic-pharmacodynamic modelling in management of QT abnormalities after citalopram overdose. Intensive Care Med. 2006;32(7):1060-5.

7. Chan A, Isbister GK, Kirkpatrick CM, Dufful SB. Drug-induced QT prolongation and torsades de pointes: evaluation of a QT nomogram. QJM. 2007;100(10):609-15.

8. Ozeki Y, Fujii K, Kurimoto N, Yamada N, Okawa M, Aoki T, et al. QTc prolongation and antipsychotic medications in a sample of 1017 patients with schizophrenia. Prog Neuropsychopharmacol Biol Psychiatry. 2010;34(2):401-5.

9. Berling I, Isbister GK, Calver L, Clunas S. Digital Holter measurement of QT prolongation in ziprasidone overdose. Clin Toxicol (Phila). 2011;49(7):694-6.

10. Isbister GK, Page CB. Drug induced QT prolongation: the measurement and assessment of the QT interval in clinical practice. Br J Clin Pharmacol. 2013;76(1):48-57.

11. Berling I, Hatten BW, Hoffman RS, Othong R, Roberts DM, Mustafa RA, et al. Guidelines for reporting case studies and series on druginduced QT interval prolongation and its complications following acute overdose. Clin Toxicol. 2020;58(1):20-8.

12. Malik M. The imprecision in heart rate correction may lead to artificial observations of drug induced QT interval changes. PACE - Pacing Clin Electrophysiol. 2002;25(2):209-16.

13. Berling I, Buckley NA, Isbister GK. The antipsychotic story: changes in prescriptions and overdose without better safety. Br J Clin Pharmacol. 2016;82(1):249-54.

Publisher's Note Springer Nature remains neutral with regard to jurisdictional claims in published maps and institutional affiliations. 